\title{
Sugar-coating dendritic cells
}

\author{
In situ labeling with azido sugars enables in vivo immunomodulation of dendritic cells.
}

M etabolic glycoengineering enables the labeling of cell surface glycans with unnatural sugar analogs. These are biocompatible azido-bearing monosaccharides that can be integrated into surface glycans via the cellular machinery. This technique has been widely used in targeted cancer therapy, as well as in photothermal and photoacoustic approaches. Now, David Mooney at Harvard University and his team, including first author Hua Wang, have demonstrated that not just cancer cells but dendritic cells (DCs) as well can be metabolically labelled with azido sugars in situ. These azido sugars can then be conjugated to fluorescent dyes in a click chemistry reaction, to enable DC tracking in vivo. DCs are professional antigen-presenting cells and crucial to the adaptive $\mathrm{T}$ cell response. "There is limited technology to track autologous dendritic cells in vivo without first having to manipulate [them] in vitro. The idea to do this came from my previous experience in metabolically labeling cancer cells," says Wang.

The researchers labeled DCs in vivo by trapping azido sugar nanoparticles inside a porous alginate gel and injecting the gel scaffold into wild-type mice. The gel was also loaded with GM-CSF, a crucial cytokine for DC recruitment, to enable a controlled release of the cytokine. This ensured that DCs would migrate to the site of the gel in response to the cytokine gradient. When the DC recruitment reached its peak, the gel was disrupted via ultrasound to release the sugar nanoparticles trapped inside, allowing cells in the vicinity to metabolize the azido sugars. Since azido labeling is not cell-type specific, the researchers had to avoid background noise due to non-specific labeling. "Among the various biomaterials we had developed in the lab, we chose one that recruited about $80 \%$ DC population," explained Wang. The remaining $20 \%$ of the recruited cells consisted of neutrophils and macrophages, which because of their short lifespans and inability to migrate to lymph nodes, respectively, did not interfere with DC tracking.

To ensure that the azido sugars were not lost from the gel through passive diffusion,

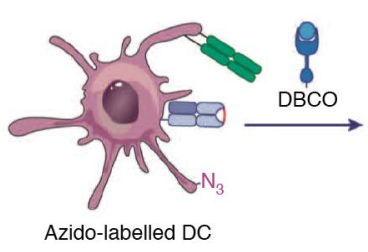

CD80/86

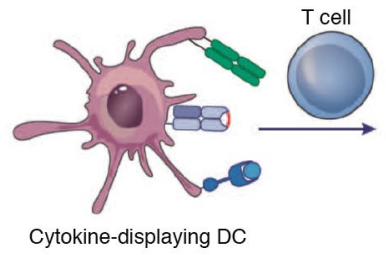

Cytokine-displaying DC

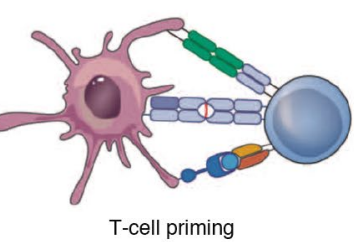

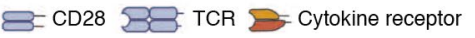

DBCO-modified cytokines bind to the azido groups and enhance T cell priming. Reprinted with permission from H. Wang et al. Nat. Mater. doi:10.1038/s41563-020-0680-1, Springer Nature.

thereby interfering with GM-CSF-mediated DC recruitment to the site, the researchers polymerized the azido sugars to generate chains of about 400 units. According to Wang, this process was the most challenging part of the study. "We wanted to make the gel perfect, so it wouldn't gradually leak the azido sugars. We wanted to wait a few days before releasing the sugars, since that is when we get the maximum DC recruitment of about one to two million cells," emphasized Wang.

To track DC migration to lymph nodes, the researchers injected azido-sugar-bearing gels into the flanks of wild-type mice. Next, the researchers examined whether azido-labeled DCs in the lymph nodes could enhance T cell priming. T cell priming is the activation of naïve $\mathrm{T}$ cells upon recognition of a cognate antigen-MHC complex. To this end, the team injected mice bearing azido-DCs with fluorescently tagged and dibenzocyclooctyne (DBCO)-modified cytokines. DBCO becomes covalently linked to the cell surface azido sugars, ensuring that the DBCO-modified cytokines are displayed on the surface of the DCs in a highly specific manner. In vitro experiments involving coculture of antigen-specific $\mathrm{CD} 8^{+} \mathrm{T}$ cells with DCs displaying cytokines revealed a notable increase in $\mathrm{T}$ cell proliferation compared to that in negative controls.

Given the ability of azido-labeled DCs to capture DBCO-modified molecules, the researchers next tested the capacity of these DCs to bind DBCO-modified antigens and adjuvants in vivo. To do so, they injected mice with azido-labeled DCs with DBCO conjugated to the human papillomavirus-derived antigen E7. Typically, E7 is presented on MHC class I and induces a $\mathrm{CD} 8^{+} \mathrm{T}$ cell response. These mice were able to generate a strong anti-E7 $\mathrm{CD}^{+} \mathrm{T}$ cell response, as evidenced by $\mathrm{T}$ cell proliferation and cytokine secretion. In addition, in mice bearing $\mathrm{E}^{+}$tumors, antigen-specific $\mathrm{T}$ cells were able to eradicate the tumor, resulting in an increase in the overall survival rate.

Labeling cells with azido sugars holds promise not only for the field of immunotherapy but also in unraveling processes that govern antigen uptake and metabolism. "I am very interested in understanding why some sugars are internalized straightaway and why some persist on the cell surface for some time," explains Wang. In addition to studying mechanisms of antigen internalization, the team is now focusing on metabolically labeling $\mathrm{T}$ and $\mathrm{B}$ lymphocytes for in vivo tracking. Here, they will have to solve the problem of specificity by coming up with a method to label only the cells of interest. For a more immediate application of this method, the researchers are attempting to elicit adaptive immune responses against neoantigens for cancer immunotherapy.

Madhura Mukhopadhyay

Published online: 2 July 2020

https://doi.org/10.1038/s41592-020-0892-y

Research paper

Wang, H. et al. Metabolic labeling and targeted modulation of dendritic cells. Nat. Mater. https://doi. org/10.1038/s41563-020-0680-1 (2020). 